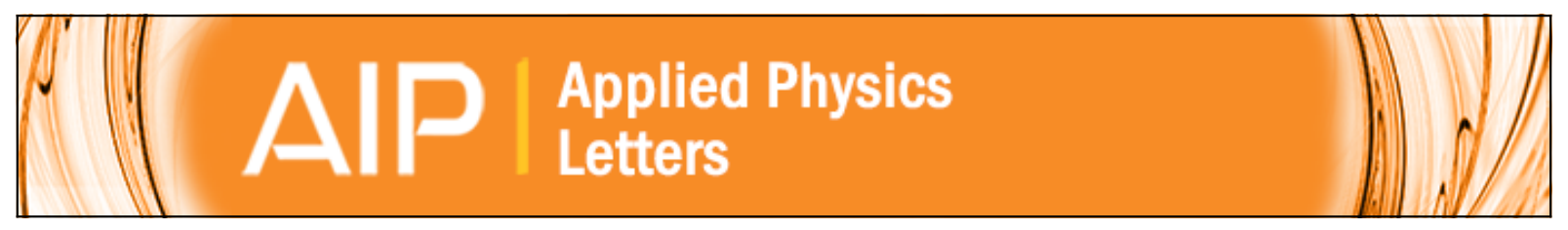

\title{
Spontaneous planarization of nanoscale phase separated thin film
}

Ravi F. Saraf, Sanjun Niu, and Eric Stumb

Citation: Applied Physics Letters 80, 4425 (2002); doi: 10.1063/1.1481187

View online: http://dx.doi.org/10.1063/1.1481187

View Table of Contents: http://scitation.aip.org/content/aip/journal/apl/80/23?ver=pdfcov

Published by the AIP Publishing

\section{Over 700 papers \&}

presentations on

multiphysics simulation visw now

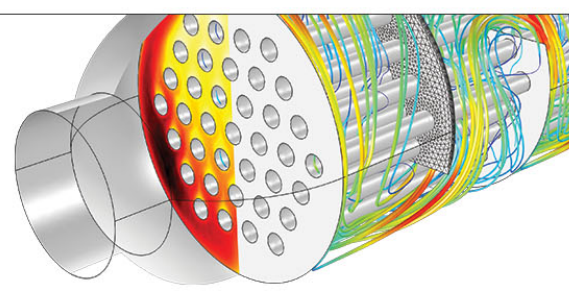




\title{
Spontaneous planarization of nanoscale phase separated thin film
}

\author{
Ravi F. Saraf, ${ }^{\text {a) }}$ Sanjun Niu, and Eric Stumb \\ Department of Chemical Engineering, Virginia Tech, Blacksburg, Virginia 24061
}

(Received 22 January 2002; accepted for publication 27 March 2002)

\begin{abstract}
Structure of complex fluid at mesoscales is influenced by interfacial effects. We describe the dynamic response in such films to sudden change in interfacial tension. In a self-assembled block copolymer film, the monolayer of $15 \mathrm{~nm}$ diam cylindrical discrete phases close to the surface commence to sink at an average rate of $0.16 \mathrm{~nm} /$ day in response to the interfacial tension change. Surprisingly, this spontaneous planarization occurs, even though the cylinders are covalently stitched to the matrix. A simple model explains the observed behavior. The observation may lead to approaches to tailor the structure of mesoscale thin films of complex fluids for long-range order that are desirable for nanoscale device fabrication. (C) 2002 American Institute of Physics.
\end{abstract}

[DOI: $10.1063 / 1.1481187$ ]

Self-assembling properties of complex fluids, such as, proteins, ${ }^{1}$ DNA, ${ }^{2}$ colloidal suspensions,${ }^{3}$ surfactants,${ }^{4}$ block copolymers, ${ }^{5}$ and liquid crystals, ${ }^{6}$ are attractive due to their potential application as template materials for (hybrid organic/inorganic) nanostructure and device fabrication. In the mesoscale regime where film thickness is comparable to the characteristic size of the building-block, i.e., the diameter of the colloid particle, or the size of the discrete phase of the block copolymer, or the radius of gyration of the protein molecule, etc., the interfacial properties have a profound effect on the structure of the complex fluid. Wetability is shown to influence the equilibrium phase segregation ${ }^{7}$ and impart topography defined by the size and shape of the discrete phase. ${ }^{8}$ In block copolymers, altering the fluid/substrate interfacial tension, the discrete phase can by oriented parallel or perpendicular to the plane of the film. ${ }^{9}$ In mesoscale polymer films, the surface enhanced mobility causes depression of the glass transition temperature ${ }^{10}$ and significant increase in crystalline order. ${ }^{11}$ Furthermore, the self-assembled structures of liquid crystals, ${ }^{12}$ multiphase complex fluids, ${ }^{13}$ and colloids ${ }^{14}$ can be preferentially oriented by a patterned substrate. It is thus reasonable to expect that the self-assembled structure of complex fluids at mesoscale film thickness will respond to dynamical changes in interfacial property that may occur due to, say environmental factors, external fields, or subsequent deposition of foreign matter. The understanding of such (dynamical) effects may lead to avenues to tailor self-assembly processes for applications such as, nanostructure fabrication.

In this letter, we report, the dynamic response to sudden change in interfacial tension in a $57 \mathrm{~nm}$ thick block copolymer film composed of a monolayer of $15 \mathrm{~nm}$ diam phase separate cylindrical phase. Surprisingly, the cylinders commence to sink due to the change in air/film environment, even though the phases are stitched together with a covalent bond and the cylindrical phases are vitrified. The spontaneous planarization process initiated due to such a change in surface tension contrasts with numerous atomic force mi-

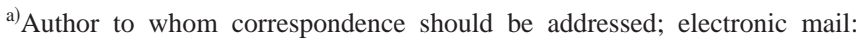
rsaraf@vt.edu
}

croscopy (AFM) studies on similar mesoscale systems where topography conforming to the underlining block copolymer structure is obtained. ${ }^{15}$ The observed dynamic behavior indicates that significant flow can occur in mesoscale thin films due to surface forces.

Figures 1(a) and 1(b) shows a height and phase image of a solvent cast polystyrene-polyisoprene-polystryene (SIS) triblock copolymer obtained by an AFM. The molecular weights of each polystyrene (PS) and polyisoprene (PI) block are 18000 and $64000 \mathrm{~g} / \mathrm{mole}$, respectively, with polydispersity of $<1.09$. Thin film is prepared by spin coating a $1 \%$ toluene solution of SIS on the Si substrate. The film is

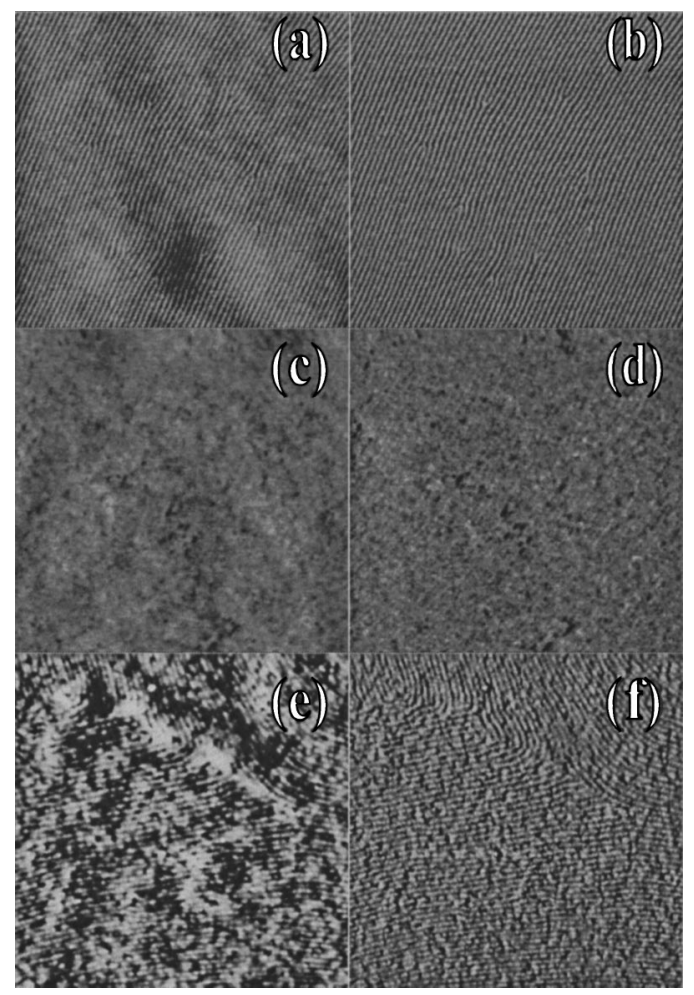

FIG. 1. AFM images of the surface topography [i.e., height image (left side)] and surface hardness [i.e., phase image (right side)] of SIS sample after the solvent annealing process. The lighter regions at higher elevation/ hardness are PS cylinders. The sample is the same, and the area imaged varies in each set. Each image is 2 by $2 \mu \mathrm{m}$. 
then dried for $150 \mathrm{~min}$ in a nominally sealed vessel under toluene vapors well below saturated vapor pressure. ${ }^{16}$ The thickness of the film after solvent annealing, measured by $\mathrm{x}$-ray reflectivity is $57 \mathrm{~nm}$. The thickness remains constant over 1 month, indicating insignificant residual solvent. The image in Fig. 1(a) reveals long-range order of polystyrene cylinders. The apparent height of the cylinders immediately after solvent annealing (i.e., at aging time, $t=0$ ) is 2.44 $\mp 0.10 \mathrm{~nm}$ and the intercylinder distance is $29 \mathrm{~nm}$.

Subsequently, the film is allowed to age at $25^{\circ} \mathrm{C}$ under ambient air. The morphology is monitored by AFM. The height images in Figs. 1(a) and 1(c) indicates that after $314 \mathrm{~h}$ of aging, the cylinders disappear in the height image. However, in the phase image in Fig. 1(d) they are still observable. Thus, the cylinders have sunk but the softer PI layer is not thick enough to obscure the local changes in surface hardness due to underlying PS cylinders. After 1 month of aging, the surface appears fully planarized with no apparent structure in height and phase image. However, after reactive ion etching of a $314 \mathrm{~h}$ aged sample, the "fingerprint" pattern reappears in both height and phase images [Figs. 1(e) and 1(f)] indicating that the cylinders are imbedded in the sample.

The average amplitude of the undulation due to the cylinders $h$ [defined in Fig. 3(a)] at a given aging time $t$ is obtained by measuring the topography over $<380$ cylinders from six images with eight cross sections per image. Figure 2(a) shows $1 / h^{2}$ versus $t$ behavior. The error bar is the standard deviation over the $<380$ points. [The rational for plotting $1 / h^{2}$ rather than $h$ is to make a comparison with the theory discussed subsequently in Eq. (1) more apparent.] Two features emerge: (i) The sinking is over $2 \mathrm{~nm}$ in $300 \mathrm{~h}$ or an average sinking rate of $0.16 \mathrm{~nm} /$ day. (ii) The reduction of $h$ [i.e., increase in $1 / h^{2}$ in Fig. 1(a)] is gradual before $70 \mathrm{~h}$ followed by an abrupt increase at $t=70$ and $106 \mathrm{~h}$.

The planarization phenomena can be explained as an interfacial behavior. ${ }^{17}$ In the solvent-annealing chamber the film is in contact with a air/toluene mixture that has higher surface tension than air. As the film is brought out of the chamber to toluene-free air, the film/air interfacial tension abruptly increases (i.e., step function). The Helmholtz free energy of the film will reduce by decreasing the surface area, i.e., "dampening" the surface undulation caused by the cylinders. The undulations can be dampened by increasing the thickness of the surface layer by diffusing more PI from the "bulk" to the surface.

Although the PI chains are (topologically) constrained by the two PS chain ends imbedded in the cylinder, it is not surprising to expect such a (high activation energy) process. A rationale for such a diffusion process to occur is the fact that the PS chains in the cylinders are not significantly entangled. ${ }^{18}$ However, as the process continues to evolve, the deformation energy stored in the cylinder due to this PI diffusion process will become significant. The stored energy is eventually released by breaking the cylinders. This fracturing process of the cylinder seems to manifest as steps in the aging curve shown in Fig. 2(a): up until $t=70 \mathrm{~h}$, defined as the "early aging-period" in the aging curve, the cylinders are continuous as seen in Figs. 2(b) and 2(c). The stripes become discontinuous in Fig. 2(d), indicating that the cylinders com-
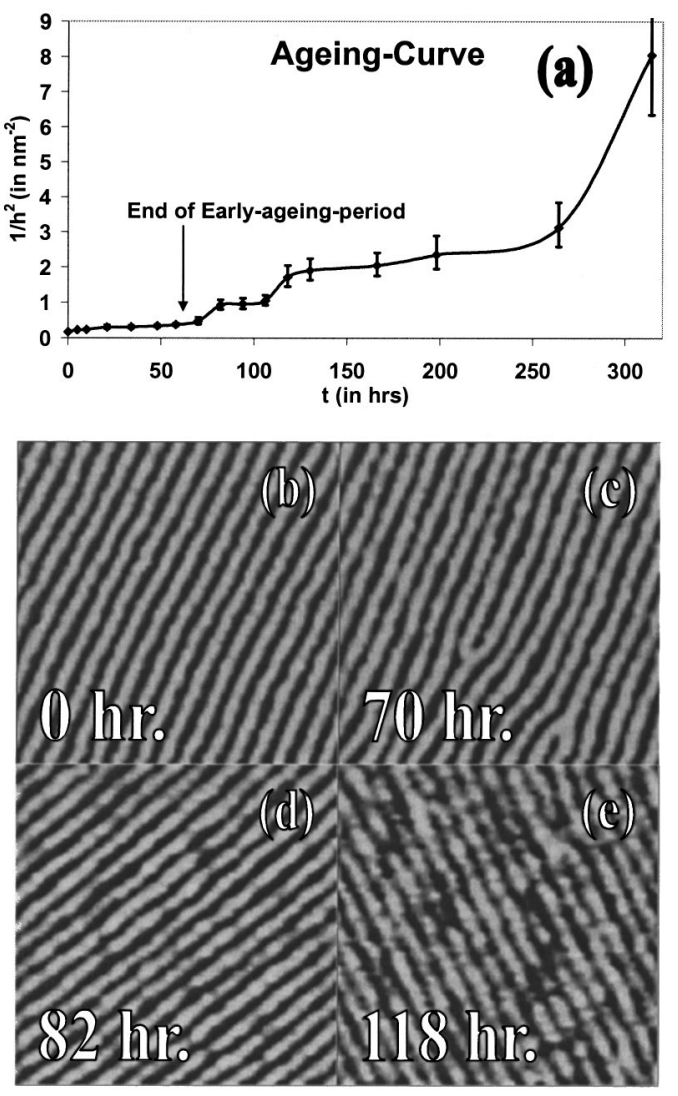

FIG. 2. (a) The aging curve plotted as $t$ vs $1 / h^{2}$ shows three steps at $70-82$ $\mathrm{h}, 106-118 \mathrm{~h}$, and $264-314 \mathrm{~h}$. The $h$ is averaged over $>380$ undulations over PS cylinders. The error bars around $1 / h^{2}$ are asymmetric [more clearly visualized in Fig. 3(b)] because the error is defined as the deviation with respect to average $h$. (b)-(e) are 500 by $500 \mathrm{~nm}$ phase images at the aging times indicated. The lighter stripes are PS cylinders.

mence to break for $t>70 \mathrm{~h}$. The number of breaks in the cylinders further increase at $t=118 \mathrm{~h}$ as seen in Fig. 2(e) compared to $t=82 \mathrm{~h}$. This is consistent with the second abrupt change in sinking kinetics seen in Fig. 2(a). The cylinders continue to break, gradually leading to morphology similar to Figs. 1(d) or 1(f).

In the initial stages of the planarization process (i.e., for $t \leqslant 70 \mathrm{~h}$ ) where the deformation effect may be ignored, the chemical potential driving the PI diffusion can be calculated by a simple model that assumes a PI surface layer that covers the "bulk" PI matrix and the PS cylinders. The existence of such a surface layer (well known in the literature) is because PI has a lower surface energy than PS, therefore the film minimizes the total free energy by forming such a layer at the film/air interface. Figure 3(a) shows a schematic diagram of the structure and the surface layer of thickness $\xi$. As the PI chains move toward the surface, $\xi$ will increase. Since the surface layer undulations "damp" with larger $\xi, \Delta h / \Delta t<0$. Change in free energy, $\delta F=\sigma \delta A=\sigma w \delta s$, where $\delta A$ is change in surface area, $\sigma$ is the (change in) air/PI surface tension, $w$ is unit depth (in the $z$ direction), and $s$ the contour length per period $\lambda$ of the surface (in $x$ direction). Thus, the chemical potential difference between the surface layer and matrix PI, $\Delta \mu=\delta F / \delta m$, where the mass diffusion $\delta m$ $=\rho w[\delta(\xi s)]$. To obtain the scaling relationship, we assume: (i) a constitutive equation, $h(\xi)=\left(R^{2} / \kappa\right) \exp (-\xi / \kappa)$, where $R$ is nominally the radius of the cylinder and $\kappa$ is like a "per- 

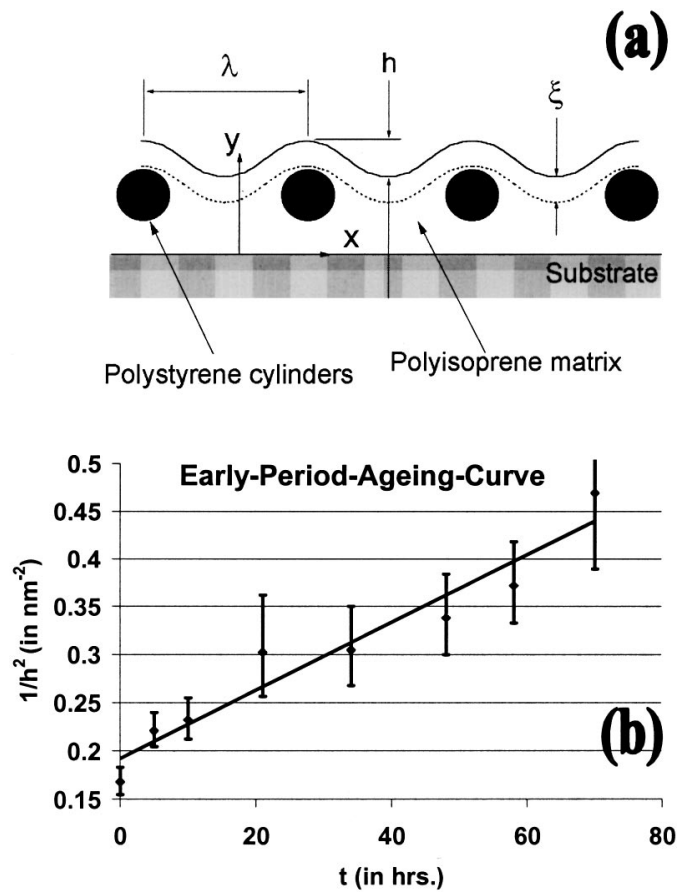

FIG. 3. (a) A schematic of the block copolymer monolayer with cylinders along the $z$ axis and aligned parallel in the $x$ axis direction. The period and amplitude of undulations due to the cylinders are $\lambda$ and $h$. By AFM, $\lambda$ $\sim 29 \mathrm{~nm}$ and $h \sim 2.44 \mathrm{~nm}(t=0)$ to $0.35 \mathrm{~nm}(t=314 \mathrm{~h})$. The permeable PI surface layer of thickness $\xi$ is defined at the air/film interface with the dotted line nominally tangent (and conformal) to the cylinder. (b) The aging behavior replotted from Fig. 2(b) to indicate the $t$ vs $1 / h^{2}$ linear behavior in a $t=0-70 \mathrm{~h}$ period, predicted by Eq. (2).

sistence length" of the surface layer that will depend on its stiffness, ${ }^{19}$ and (ii) a triangular function to model the surface contour, leading to $s(h)=\lambda\left\{1+(2 h / \lambda)^{2}\right\}$. The chemical potential then becomes $\Delta \mu=-(8 \sigma / \rho \kappa)\left(h^{2} / \lambda^{2}\right)$.

By assuming a linear profile for $\Delta \mu$, the diffusion flux ${ }^{20}$ of PI toward the surface becomes $j=L[\Delta \mu / \xi]$ $=(w \lambda \rho)^{-1} \delta m / \delta t . L$ is proportional to Onsager's mobility. Using the $h(\xi)$ and $s(h)$ functions, and integrating, the flux equation yields

$$
\frac{1}{h^{2}}=\frac{1}{h_{0}^{2}}+\frac{32 L \sigma}{\rho \lambda^{2} \kappa^{3}} t
$$

where $h_{0}=h(t=0)$. Figure 3(b) shows a good agreement between the experimental data and $t$ versus $h$ dependence predicted by Eq. (2) for early stage aging (i.e., $t \leqslant 70 \mathrm{~h}$ ).

The significant flow in film due to change in surface tension where PS cylinders are vitrified indicates the dominance of the constraints imposed by interfaces. Such interfacial constraints caused by surface layer geometry and (chain) topology will perturb the long-range order in the thin film monolayer structure. In thermal annealing, the mobility to annihilate defects is increased, ${ }^{21}$ leading to better order, but the constraint to maintain an equilibrium surface layer is unchanged. This partially explains the observation of significantly longer-range order in solvent-annealed film compared to films made by thermal annealing. ${ }^{22}$ Thus processes that will diminish the interfacial constraints will improve longrange order.
R.F.S. would like to thank Carilion Biomedical Institute, VA, Optical Science and Engineering Center, Virginia Tech, and IBM's T. J. Watson Research Center, NY for financial support.

${ }^{1}$ C. M. Niemeyer, T. Sano, C. L. Smith, and C. R. Cantor, Nucleic Acids Res. 22, 5530 (1994); E. Winfree, F. Liu, L. A. Wenzler, and N. C. Seeman, Nature (London) 394, 539 (1998).

${ }^{2}$ Saghatelian, Y. Yokobayashi, K. Soltani, and M. R. Ghadiri, Nature (London) 409, 797 (2001); O. Rathore and D. Y. Sogah, J. Am. Chem. Soc. 123, 5231 (2001).

${ }^{3}$ S. Mann and G. A. Ozin, Nature (London) 382, 313 (1996); N. Pinna, K. Weiss, J. Urban, and M. P. Pileni, Adv. Mater. 13, 261 (2001).

${ }^{4}$ W. F. Muller, D. L. Klein, T. Lee, J. Clark, P. L. McEuen, and P. G. Schultz, Science 268, 272 (1995); R. Maoz, E. Frydman, S. R. Cohen, and J. Sagiv, Adv. Mater. 12, 424 (2000).

${ }^{5}$ Y. Lee, N. Yao, and I. A. Aksay, Langmuir 13, 3866 (1997); C. T. Blake, K. W. Guarini, K. R. Milkove, S. M. Baker, T. P. Russell, and M. T. Tuominen, Appl. Phys. Lett. 79, 409 (2001).

${ }^{6}$ P. V. Braun, P. Osenar, V. Tohver, S. B. Kennedy, and S. I. Stupp, J. Am. Chem. Soc. 121, 7302 (1999).

${ }^{7}$ R. A. L. Jones, L. J. Norton, E. J. Kramer, F. S. Bates, and P. Wiltzius, Phys. Rev. Lett. 66, 1326 (1991).

${ }^{8}$ R. F. Saraf, J. Roldan, and T. Derderian, IBM J. Res. 38, 441 (1994); P. Keblinski, S. K. Kumar, A. Maritan, J. Koplik, and J. R. Banavar, Phys. Rev. Lett. 76, 1106 (1996).

${ }^{9}$ P. Mansky, Y. Liu, E. Huang, T. P. Russell, and C. Hawker, Science 275, 1458 (1997).

${ }^{10}$ W. J. Orts, J. H. Vanzanten, W. L. Wu, and S. K. Satija, Phys. Rev. Lett. 71, 867 (1993); L. Xie, G. B. DeMaggio, W. E. Frieze, J. DeVries, D. W. Gidley, H. A. Hristov, and A. F. Yee, Phys. Rev. Lett. 74, 4947 (1995).

${ }^{11}$ B. J. Factor, T. P. Russell, and M. F. Toney, Phys. Rev. Lett. 66, 1181 (1991); R. F. Saraf, C. Dimitrakopoulos, M. F. Toney, and S. P. Kowalczyk, Langmuir 12, 2802 (1996).

${ }^{12}$ W. M. Gibbsons, P. J. Shannon, S. T. Sun, and B. J. Swetlin, Nature (London) 351, 49 (1991); M. Ibn-Elhaj and M. Schadt, ibid. 410, 796 (2001).

${ }^{13}$ M. Boltau, S. Walheim, J. Mlynek, G. Krausch, and U. Steiner, Nature (London) 391, 877 (1998); L. Rockford, Y. Liu, P. Mansky, T. P. Russell, M. Yoon, and S. G. P. Mochrie, Phys. Rev. Lett. 82, 2602 (1999).

${ }^{14}$ K. H. Lin, J. C. Crocker, V. Prasad, A. Schofield, D. A. Weitz, T. C. Lubensky, and A. G. Yodh, Phys. Rev. Lett. 85, 1770 (2000); Y. H. Ye, S. Badilescu, V. V. Truong, P. Rochon, and A. Natansohn, Appl. Phys. Lett. 79, 872 (2001).

${ }^{15}$ See, for example, J. T. Chen and E. L. Thomas, J. Mater. Sci. 31, 2531 (1996).

${ }^{16}$ The film preparation process is similar to: G. Kim and M. Libera, Macromolecules 31, 2569 (1998).

${ }^{17}$ Placing the film after the solvent annealing on a hot plate at $80{ }^{\circ} \mathrm{C}$ for 30 min in $\mathrm{N}_{2}$ environment to remove the residual toluene leads to no significant change in surface morphology observed by AFM. Subjecting the solvent-annealed film to $50 \mathrm{mTorr}$ pressure at $25^{\circ} \mathrm{C}$ for $120 \mathrm{~min}$ also had no change on the morphology. Thus the sinking process is not due to residual solvent evaporation.

${ }^{18}$ The entanglement-molecular weight for PS is $\sim 18000$, see J. D. Ferry, Viscoelastic Properties of Polymers, 3rd ed. (Wiley, New York, 1980), p. 374, Table 13-1.

${ }^{19}$ As $\xi$ increases, $h$ should decrease such that $h \rightarrow 0$ as $\xi \rightarrow \propto$ and $h$ is maximum at $\xi=0$. Thus, $h=q(\xi)=\left(R^{2} / \kappa\right) \exp (-\xi / \kappa)$, is a reasonable constitutive equation for $\xi<\kappa$ (a reasonable assumption for the studied geometry): $h(\xi)$ will decrease as $\kappa$ or/and $\xi$ increases.

${ }^{20}$ S. R. de Groor and P. Mazur, Non-Equilibrium Thermodynamics (Dover, New York, 1984), p. 229.

${ }^{21}$ C. Harrison, D. H. Adamson, Z. D. Cheng, J. M. Sebastian, S. Sethuraman, D. A. Huse, R. A. Register, and P. M. Chaikin, Science 290, 1558 (2000).

${ }^{22}$ For the similar system studied, significantly poor order, attributed to interfacial effects, is observed for the thermal annealing process, see P. Mansky, P. Chaikin, and E. L. Thomas, J. Mater. Sci. 30, 1987 (1995). In our studies, SIS films annealed for $2-12 \mathrm{~h}$ at $120^{\circ} \mathrm{C}$ in $\mathrm{N}_{2}$ environment are significantly more twisted than solvent annealed films for $150 \mathrm{~min}$. The nominal persistence lengths of the cylinders in annealed and solventannealed film are $\sim 500$ and $>3000 \mathrm{~nm}$, respectively. 